\title{
Maintaining Communication Links Using a Team of Mobile Robots
}

\author{
W. Zhuang, X. Chen, J. Tan
}

\author{
Wei Zhuang \\ Nanjing University of Information \\ Science \& Technology \\ Nanjing,210044,P.R.China \\ E-mail: zw@nuist.edu.cn \\ Xi Chen, Jindong Tan \\ Michigan Technological University \\ Houghton,MI 49931,USA \\ E-mail: xchen@mtu.edu, jitan@mtu.edu
}

\begin{abstract}
:
This paper presents a comprehensive metric to evaluate the link quality and the corresponding control schemes for the distributed control of a team of robots to maintain the communication links. The mobile robots dynamically reconfigure themselves to maintain reliable end-to-end communication links. Such applications require online measurements of communication quality in real time and require a mapping between link quality and robot positions. In this paper, we present the empirical results and analysis of a link variability study for an indoor and outdoor environments including received signal strength indicator (RSSI), throughput and packet loss rate. The distributed control algorithms consider the environmental constrains and obstacles. Moreover, the self-deployment algorithms allow a team of robots to recognize the coverage gap by monitoring link qualities, and deploy the mobile robots for a variety of applications including self-healing, tethering, intelligent relaying. The assessment of link quality acts as the feedback for cooperative control of mobile robots. The experimental results have shown the effectiveness of evaluation for communication links and the related control schemes.
\end{abstract}

Keywords: Communication Link, Networked Robots, Decentralized Control, RSSI.

\section{Introduction}

With the advancement in wireless sensor technologies, a communication network can be rapidly setup by densely deploying sensor nodes in an area of interests. These intelligent wireless sensors will self-organize themselves to form a communication and sensor grid. The quality of communication links are subject to many factors including the distance between neighboring nodes, the topology of the network, the environment attenuation, and the use of battery. The wireless sensors, once deployed, can not be reconfigured to maximize the network performance nor to guarantee end-to-end data transmission. The failure of a few wireless nodes on a critical path may lead to the failure of the entire network. There are many advantages to use mobile robots to setup a temporary communication network whose link qualities can be dynamically maintained by the motion control of mobile robots.

Fig. 1 shows a scenario where a team of mobile robots can be used to setup a self-organizable communication network in battlefield. These robots can be equipped many sensors for monitoring and reconnaissance of battlefields. Military personnel relies on these sensors for situation 


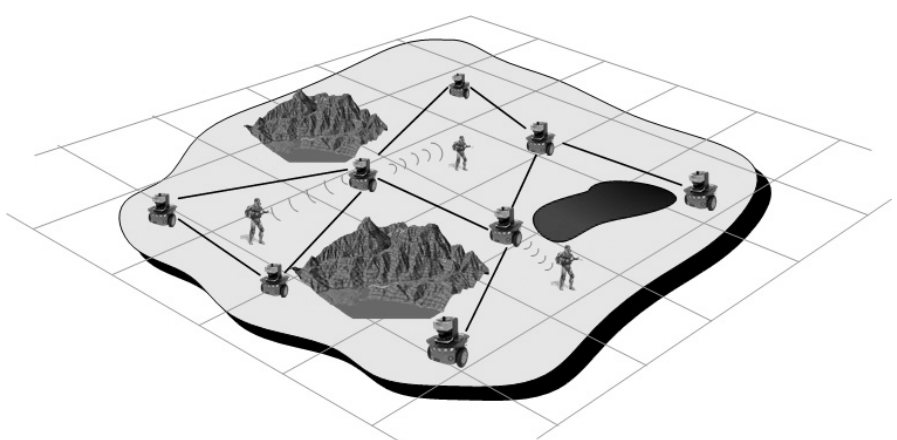

Figure 1: A large scale battlefield cooperation using networked robotic team

awareness. In many circumstances, communication connections could be lost due to many reasons, such as ubiquitous wireless signal noise, angle of signal arrival, natural obstacles, and even a slight movement of pedestrian. The natural settings such as mountains and lakes shown in Fig. 1 represents some of the barriers for smooth communication traffic within the network. In these situations, neighboring mobile robots can monitor their link quality and re-position themselves accordingly to the measurement of link quality. A rapidly deployable mobile network with a team of mobile robots has many potential applications in military and disaster relief.

The self-deployment and maintaining a team of mobile robots face challenges in both link quality measurement and distributed control a multi-robot system. The quality of an end-end communication link are affected by both many factors including the ambient noise, environment attenuation, battery level, motion of the robots, direction of the antenna, etc. Many evaluation metrics such as signal to noise ratio, received signal strength, packet loss, etc. None of these evaluation metrics are easily quantified for control purposes. The second challenge is the distributed control of mobile robots for dynamic link quality improvements. Though distributed control of multiple mobile robots have been intensively discussed, the cooperative control of mobile robots for communication links faces new challenges in the coupling of environment and communication factors.

The monitoring of communication links has long been a research topic in the communication society, and many approaches for evaluating link quality have been proposed. It is shown that that distance cannot be directly adopted as an assessment for link quality [1]. Base on the celebrated Shannon-Hartley theory, the capacity of communication link is proportional to SNR (signal-to-noise ratio). Souryal et al. validated that SNR can reliably predict the link quality. However, it is difficult to improve the SNR measurements with this model due to consideration of external interference in the model [2]. Dixon and Frew employed SNR of the communication links to define an optimal communication chain [3]. Apart from using SNR to evaluate link quality, researchers adopted wireless signal strength to assess the quality of connectivity in multi-robot system. Luthy and Grant et al. used RSSI measurements to repair disconnected wireless sensor networks [4]. Stump et al adopted Fielder value (the second-smallest eigenvalue) to describe the degree of connectivity in a communication grid [1]. Packet loss rate can also serve as a metric to reflect the performance of communication links. In this work, we analyze a set of comprehensive metrics ,RSSI, throughput, packet loss rate and velocity,to explore the possibility of controlling mobile robots for reliable end-to-end communication links. RSSI measurement may serve as an main metric to assess the link quality. However, from the results of our experiments, some other metrics such as speed, angle of arrival, can also reflect the link quality.

Many control algorithms for a large distributed robotic cooperation are presented before. These approaches could be divided into two categories, the centralized and decentralized algo- 
rithms. Generally, the question of whether to have centralized or decentralized control comes down to resources [5]. If one of the robots is defined as a central unit which is in charge of the data fusion and decision, the entire system could be brittle when such the manager did not work [6]. Additionally, communication overhead and response time are limiting factors for centralized control. In our previous work, a scalable graph model for decentralized control of mobile robots was discussed. The combination of Delaunay triangulation and potential field make it possible to adjust the robot positions in a distributed way while a global objective is achieved [7]. Similarly, a decentralized mobility control scheme using Extremum Seeking method was presented to form a linked chain of mobile relays [8]. They focused on maximizing the total link bandwidth. Simulation results and movement data from different platforms performing different applications verified the above approaches [9]. In this work, we discuss the control of multiple mobile robots to guarantee reliable end-to-end communication links using a distributed control method.

\section{Impact of Comprehensive Metrics}

In this section, we introduce the analysis of three metrics and related effects on communication links, RSSI (Received Signal Strength Indicator), throughput and packet loss rate respectively. The Shannon-Hartley theory stated that the channel capacity $C=B \log _{2}(1+S / N)$. Where, $C$ is the channel capacity, and $B$ is the bandwidth of the channel. $S$ is the total signal power over the bandwidth, and $N$ represents the total noise power over the bandwidth. With a fixed noise distribution and bandwidth, the metric $S$ is proportional to channel capacity which denoted RSSI should be adopted as a metric to evaluate the links. Throughput as a conventional metric to define quality of end-end communication link is also taken into account. Moreover, packet loss rate, a real-time traffic steam detector, is accepted as a threshold to estimate network health status. We take these metrics into account so that the quality of link could be evaluated qualitatively and quantitatively.

\subsection{Received Signal Strength Indicator (RSSI)}

\section{RSSI VS. Distance}

In wireless communication area, Received Signal Strength Indicator (RSSI) is a measurement of the power present in a received radio signal. RSSI measurements from mobile sensor networks could be directly acquired using simple light-weight processing, especially in IEEE 802.11 protocol family. Generally, RSSI is not a stable measurement owning to the multi-path fading and shadowing [10]. It is not linearly proportional to corresponding physical distance. However, it could be considered as a threshold to evaluate the quality of link. Fig. 2(a) shows the ubiquitous fluctuation of RSSI measurement when the robot is deployed in static. We set the distance between a sender and a receiver as 2 meters in a noisy corridor. Two sets of experiments have been done in a noisy corridor and open space outside respectively. It is obvious that the measurements sampled in outdoor are larger than that in a constrained indoor environment. The figure shows the measurements fluctuating all the time. The largest amplitude of measurements reaches $-5 \mathrm{dBm}$. Another set of experiments aims to test feasibility of RSSI as a metric for evaluating links in the same two scenarios. Fig. 2(b) shows RSSI value varies with the movement of mobile robots. One is set to move forward along a straight line. The results illustrates RSSI values varies synchronously with corresponding distance. In a large scale area, if the noise distribution is fixed, we can adopt RSSI value as a metric to reflect the link quality inferred from the Shannon-Hartley theory. 

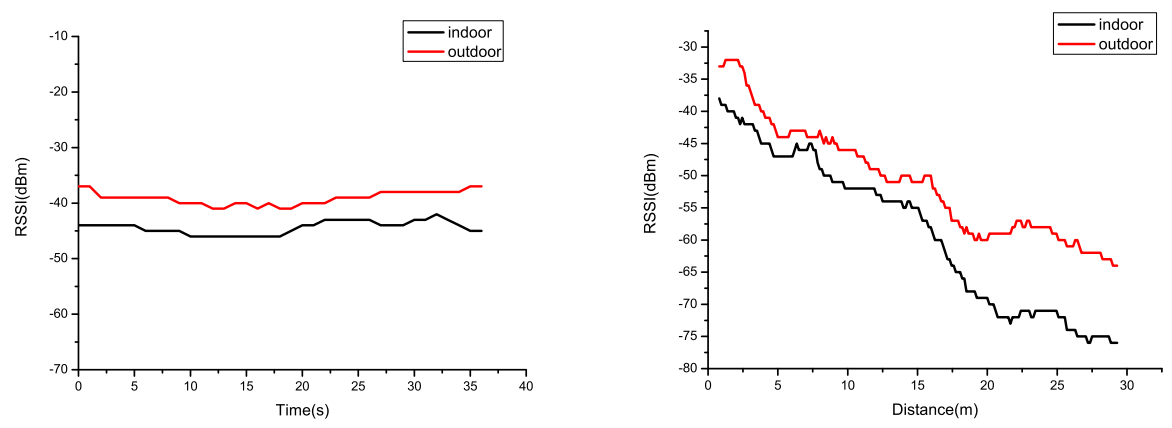

(a) Fluctuation of RSSI in static(indoor and (b) RSSI VS. distance during the movement outdoor) of robot

Figure 2: RSSI performance with and without mobility

\section{Angle of Arrival}

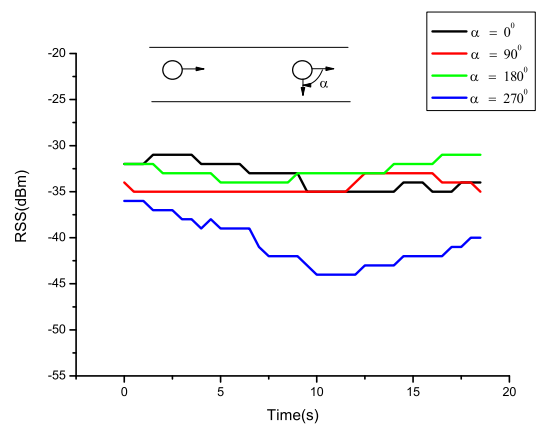

(a) RSSI performance with robots being deployed in a line

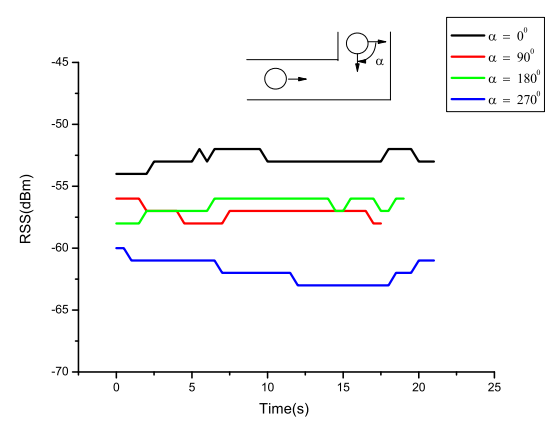

(b) RSSI performance with robots being deployed in a diagonal

Figure 3: Impact of angle of arrival

It have been testified that the angle of arrival could affect the performance of RSSI measurements [11]. In this section, we also illuminate this phenomenon during the movement of robots. Theoretically, the antenna of $802.11 \mathrm{~b} / \mathrm{g}$ compatible network device is omni-directional, that is, no matter what direction it receives from the signal, the RSSI measurers should be the same. But, these off-the-shelf devices do not show the symmetric feature in different orientation. Fig. 3(a) and Fig. 3(b) shows the variety in different signal received directions when being deployed in a line and diagonal respectively. Two robots are deployed in a long corridor, where one is in static and the other is moving. The robot changes its orientation clockwise in turn at the same spot. Both of the results show that RSSI value has the minimum when the angle turns to 270 degree. The difference of measurements is up to $-10 \mathrm{dBm}$. Moreover, the RSSI difference between 0 degree and the other degrees is larger than that in a line. The results can be useful for the reliable sampling of RSSI since the angle between neighbor robots could varied during the task.

\section{Speed}

Speed is also concerned both in RSSI and throughput performance. Note the speed we mentioned here is the velocity of mobile robot rather than signal modulation rate. In order 
to obtain the real influence on the performance in mobile sensor network, we set up a series of outdoor experiments to testify it. Two mobile robots equipped with $802.11 \mathrm{~b} / \mathrm{g}$ compatible network devices are deployed on a long road. We selected the maximum translate velocity of the robots as $700 \mathrm{~mm} / \mathrm{s}$ and the minimum speed as $100 \mathrm{~mm} / \mathrm{s}$. To avoid the interference of other metrics like angle of arrival, etc., one of the robots is requested to move straightly, the other was stationary. The final trajectory is a straight line.

Fig. 4(a) shows the connection between RSSI and related distance. The black curve (upper line) and the red curve(lower line) denoted the relationship with the speed of $100 \mathrm{~mm} / \mathrm{s}$ and $700 \mathrm{~mm} / \mathrm{s}$ respectively. It was obvious that the RSSI measurements with lower speed is always larger than that with higher speed at the same distance except for the startup period. Note that the convex curve of the red line was shown in Fig. 4(a). Theoretically, the curve is proportional to $\lambda^{-n}$ where $\lambda$ is distance, and presents monotonic decreasing. However, with higher speed, the RSSI measurements could be affected seriously by multi-path fading and reflection of constructions. Here, the convex part locates at a special zone in which RSSI could be disturbed acutely. Meanwhile, the difference between fixed RSSI value with two speed modes fluctuated rather than increasing continuously which is shown in Fig. 4(b). These could be also explained by the special zones the robot passed in and out. Here, the results illuminate that at a higher speed the robot moved, the much more possibility of interference and instability of RSSI occurred.

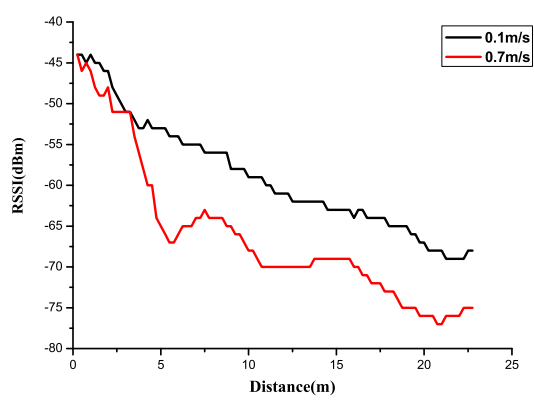

(a) Impact of translate velocity on RSSI

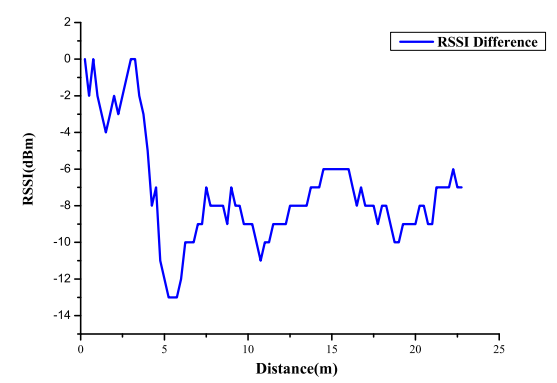

(b) RSSI difference of two speed modes

Figure 4: RSSI vs. distance with different speeds

\subsection{Throughput performance}

In this section, we introduce the performance of throughput in our proposed environment. Mobility, transmission rate and speed of robots were concerned. Throughput as a conventional metric in communication network reflects the performance of communication capacity [12]. As we mentioned before, if the robot moved into a "gray zone" [13] or extremely noisy area, the measurements of RSSI could decayed by a small value $(\mathrm{dBm})$ but the link is broken possibly. So, throughput and related parameters like response time or latency are prone to be accepted as compliments for the entire evaluation.

\section{Mobility}

Compared with computer networks, mobility is one of emphasis in mobile sensor network which can have effects on the throughput of neighbor robots. We deployed two mobile robots (Pioneer-3AT) in a walled indoor environment. One is set in static, the other moves along the corridor. IxChariot, a Tcp/Udp packets generator and analyzer is used to obtain real time 


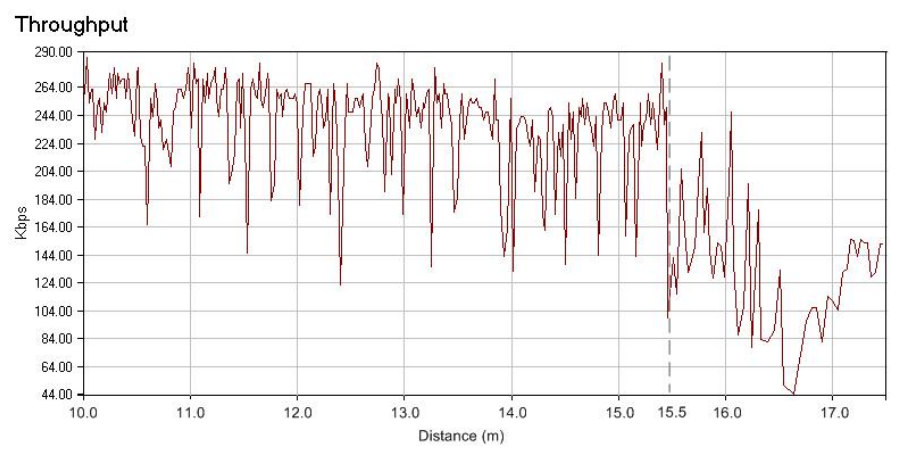

Figure 5: Results of active measurements during the movement

Table 1: Ixchariot parameters of mobility

\begin{tabular}{|c||c|}
\hline Console Protocol & TCP \\
\hline Scripts & Credits \\
\hline number of timing records & 50 \\
\hline size of record to send & 100 \\
\hline send buffer size & Default \\
\hline
\end{tabular}

throughput in our experiments. All the network parameters are listed in Table 1. Fig. 5 shows the fading of the throughput with movement. The obvious decreasing of throughput occurred at 15.5 meters approximately. Based on the theory mentioned above, as long as the mobile robot stays in a circle with the radius less than a value the communication link can retain connection status. If the robot moves out of the circle, it still has ability to exchange data with neighbors but the throughput presents sharply fading. Note that deploying in different scenarios (indoor and outdoor) or using different sending packets size, the metric could vary due to the multi-path fading and MAC overhead.

\section{Speed VS. Throughput}
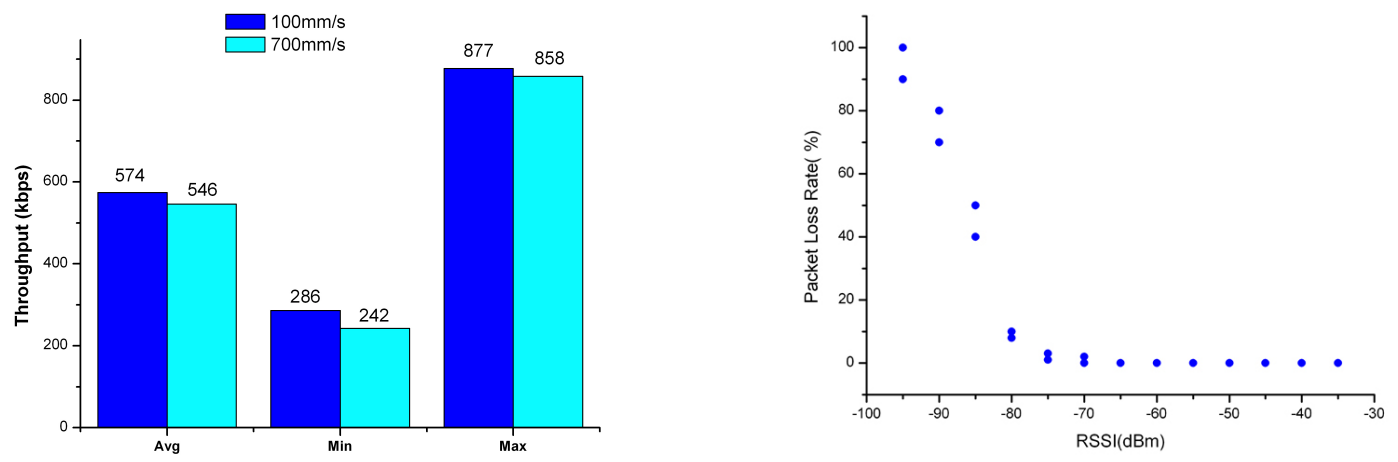

Figure 6: Comparison of throughput perfor-Figure 7: Packet loss rate VS. Received signal mance with different speeds strength

Another relationship should be concerned is the speed influence on throughput. Fig.6 shows the impact of speed on throughput. The upper line and the lower line denote transformation of 
throughput as time elapsing with the speed of $100 \mathrm{~mm} / \mathrm{s}$ and $700 \mathrm{~mm} / \mathrm{s}$ respectively. IxChariot is adopted to sample the throughput using the parameters in Table 1 . There is no obvious difference between the two lines. Since the mobile robot always keep connecting with neighbors, as long as the transmission rate stays lower than bandwidth, the traffic could be smooth and stable. The average throughput $546 \mathrm{kbps}$ at speed of $700 \mathrm{~mm} / \mathrm{s}$ is adequate for message and low-resolution low FPS video transmission.

\subsection{Packet Loss Rate}

Packet loss rate can also reflect quality of communication links in wireless network. However, [2] confirm that the low correlation between packet loss rate and distance and show the extent of variation in an indoor office environment. So, we select RSSI instead of distance as $X$ domain and corresponding packet loss rate as $Y$ domain shown in Fig.7. One robot is set to move along the corridor while the other is stationary. 100 packets ( 522 byte per packet) were transmitted from the base to mobile robot every second continuously. The RSSI interval is set as $-5 \mathrm{dBm}$, and the range is set from $-35 \mathrm{dBm}$ to $-95 \mathrm{dBm}$. The result indicated the critical area of related RSSI lay in $-85 \mathrm{dBm} \pm-5 \mathrm{dBm}$. We assumed the point A where the observed RSSI value is $-80 \mathrm{dBm}$ and point B where the sampled RSSI value is $-90 \mathrm{dBm}$. We define $d$ as the distance between A and $\mathrm{B}$. The mobile robot is arranged to move from $\mathrm{A}$ to $\mathrm{B}$ repeatedly. The average distance $\tilde{d}$ is less than 1 meter which denoted that it took less than 3.3 seconds at the speed of $300 \mathrm{~mm} / \mathrm{s}$. Generally, the interval is so short that fast responding action such as stop, turn, reverse, etc. could not be achieved in our assumed scenario. So, the packet loss rate can be fit for evaluation of data traffic integrality but not suitable for a feedback for controlling the robotic team.

\section{Decentralized Control Algorithms}

In this section, we introduce a decentralized control scheme adaptive to unconstrained and constrained scenarios. The improved RSSI measurement is adopted to be feedback input into control system. Base on the Delaunay graphic model, We define two different chained form networks describing self-healing and tethering respectively. Virtual forces composed of abstractive forces and repulsive forces are exploited to drive the robot to a stable status.

\subsection{Delaunay Triangulation and Network Model}

First, we define the graph model for the multi-robot system. The configuration and control input of the whole system could be defined as:

$$
q=\left\{q_{1}, q_{2}, \ldots, q_{n}\right\}^{T}
$$

The overall system can be denoted by $\dot{q}=f(q, u)$, where $f$ is the vector of system dynamics. In a unified inertial coordinate system, $\tilde{p}_{i}=\left\{x_{i}(t), y_{i}(t)\right\}^{T}$ is defined as the position of the robot $R_{i}$. We define $p_{i}=\left\{x_{i}(t), y_{i}(t)\right\}^{T}$ as the position of the robot $R_{i}$ in its local coordinate frame.

The Delaunay triangulation with a set of nodes is defined such that any additional edge between any two nodes intersected one of the existing edges. The Delaunay triangulation define the link properties between one-hop neighbors, which are described by a set of edges.

Here, we can adopt adjacency matrix to specify the connectivity of the Delaunay tessellation using these edges. Motivated by the connectivity in multi-robot team [3], we define the quality of communication link between $R_{i}$ and $R_{j}$ which connected with each other through one-hop link as a continuous value. This value is denoted by improved RSSI measurement. The quality is set to be: 


$$
\Psi_{i j} \triangleq \begin{cases}\Delta_{i j}, & \left\|p_{i j}\right\|<\rho \\ 0, & \| p_{i j} \mid>R \\ \exp \left[\frac{-5\left(\left\|p_{i j}\right\|-\rho\right)}{R-\rho}\right], & \text { otherwise }\end{cases}
$$

Where, $R$ means the cut-off RSSI measurement which is able to guarantee the link connection and $\rho$ means a saturation RSSI measurement where the communication status between robots does not change as they get closer together. The two parameters can be estimated by results of packet loss rate and active throughput respectively. Unlike the idea in [1],we define $\Delta$ as a variant which describes the degree of the connectivity of neighbors. All the analysis of the $\Delta$ has been demonstrated in [14], which shows the impact of comprehensive metrics including RSSI, active throughput and packet loss rate. We define an Adjacency Matrix $A(t)$ to specify the connectivity of the Delaunay triangulation. Here, $A_{i j}=\Psi_{i j}$ if $R_{i}$ and $R_{j}$ are one-hop neighbors, otherwise $A_{i j}=0$. So, the link properties of $R_{i}$ and its one-hop neighbors are denoted by $i^{t h}$ column of $A(t)$, that is $A_{i}(t)=\left\{a_{i 1}, a_{i 2}, \ldots, a_{i n}\right\}^{T}$.

\subsection{Graphic Model Based Control Algorithm}

\section{General model}

For a multi-robot system, the computation of Delaunay triangulation is time consuming. A decentralized control method is presented to calculate individually and cooperate to repair the communication link. In this paper, a distributed control method is proposed based on both the potential field method and the Delaunay triangulation. For a mobile robot $R_{i}$, a performance index (candidate lyapunov function) is defined as follows:

$$
V_{i}=\frac{1}{2} \sum_{j=1}^{N_{i}} k_{i p}\left(\left\|p_{i j}\right\|-c_{i j}\right)^{2}+\frac{1}{2} k_{i v}\left\|v_{i}\right\|^{2}
$$

Where $p_{i j}$ states the improved RSSI value between robot $R_{i}$ and robot $R_{j}$. $\left\|p_{i j}\right\|$ equals to $a_{i j}$ which is an entry in adjacency matrix $A_{i j}$, and $c_{i j}$ is the actual and desired RSSI value between the two neighboring robots. $N_{i j}$ is the total number of the one-hop neighbors of $R_{i j} . k_{i p}$ and $k_{i v}$ are the parameters for the virtual potential energy and kinetic energy of the robot. The control input is derived by:

$$
\begin{aligned}
u_{i} & =-\frac{\partial V_{i}}{\partial a_{i}}-\frac{\partial V_{i}}{\partial v_{i}} \\
& =-\sum_{j=1}^{N_{i}} k_{i p}\left(a_{i j}-c_{i j}\right) \frac{p_{i j}}{a_{i j}}-k_{i v} v_{i} \\
& =-F_{i}-k_{i v} v_{i}
\end{aligned}
$$

Here, we have obtained the necessary conditions for controlling $R_{i}$ to keep the connection. When one node is lost in communication chain, the whole system can repair the network connection using neighboring robots to fill the position automatically. If all the other robots but $R_{i}$ are static, it is easy to prove that the above controller is globally convergent [7].

\section{Self-configuration and Self-healing}

Self-healing is based on the same theory of self-configuration. In our works before, [7] proposed an control algorithm for self-configuration both in constrained and unconstrained environment. 
Fig. 8(a) shows the simulation result of self-configuration after 10 random robots implemented the cooperation scheme. Fig. 8(b) shows the result of self-healing using the general model when two nodes are disconnected with neighbors. The objective is to fill the coverage gaps the disconnected robots generated before.

\section{Tethering and Intelligent Relays}

Tethering is the reverse processing of intelligent relaying which stretches the network to be a chain as long as possible. Different with self-healing, the objective is to keep connection with neighbors so as to maximize the end-end distance. Theoretically, if the communication range is larger than double of sensing range, the chain should transform into a line in an unconstrained situation while not possible in real environment.

An control algorithm named Minimum - Follow for stretching the network is proposed in this section. The Delaunay triangulation could be broken into a chained form network. Every robot in this network just have two one-hop links at last. The nearest neighbor with respect to ready-to-move robot could be chosen to take a movement every round in the iterative loop. The offset is determined by the virtual force generated by foregoing robot. Each robot is considered to follow its foregoing moving robot. Fig. 9 shows the simulation results of tethering algorithm. We set $v_{i}$ as $1 \mathrm{~m} / \mathrm{s}$ and $c_{i j}$ as 40 . The total processing time was 100 seconds. The start-up scenario was from the stable status after self-healing shown in Fig. 8(b). As long as the space is wide enough, finally the chained form network will transform into a line. It is obvious that the cooperation using our algorithm is not optimal for stretching the network using this decentralized algorithm. However, each robot just needs to connect with neighbor robots while a powerful processing unit is able to manage the robots in centralized control.

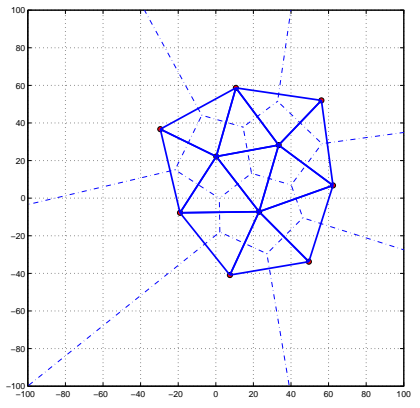

(a) Stable status of the Delaunay triangulation with 10 robots

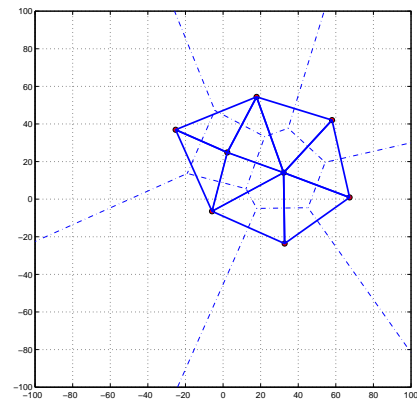

(b) Stable status with 8 robots after selfhealing

Figure 8: The simulation results of self-healing

\section{Experiments}

In this section, we introduce related experiments of indoor and outdoor to test our algorithms. We used Pioneer 3-AT as our mobile robot which is equipped with one laser detector upper the board and 8 arrays of sonar mounted in front and back flank. A Dell-D430 laptop is chosen to be the data processing unit. The real time RSSI measurements are collected through WirelessMon. In order to minimize impact of speed, the linear velocity of mobile robot is defined as $110 \mathrm{~mm} / \mathrm{s}$ and the wireless channel is set in $1 \mathrm{Mbps} / \mathrm{s}$. All the processing and control command codes are implemented in Matlab. 


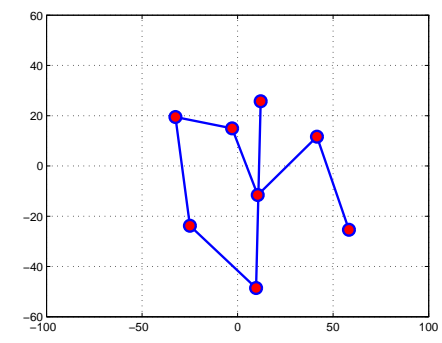

(a) The snapshot after $10 \mathrm{~s}$

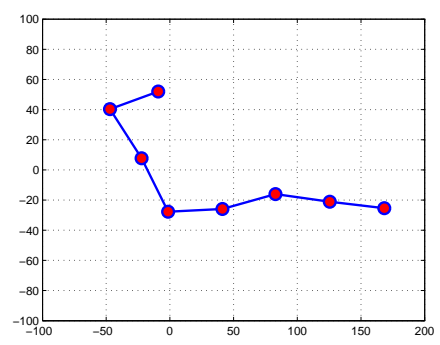

(c) The snapshot after $150 \mathrm{~s}$

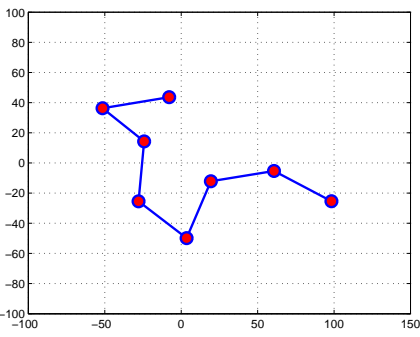

(b) The snapshot after $50 \mathrm{~s}$

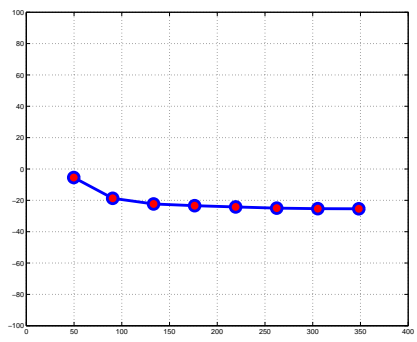

(d) The snapshot after $300 \mathrm{~s}$

Figure 9: The snapshots of tethering simulation results. The lead robot is demanded to keep moving at $1 \mathrm{~m} / \mathrm{s}$, while optional neighbor robots respond. Speeds of these ready-to-move robots are determined by virtual forces. Finally, every robot only has two one-hop neighbors in the chained form network.

Table 2: Environmental parameters of indoor and outdoor

\begin{tabular}{|c||c||c||c||c|}
\hline Environment & $\rho$ & $R$ & $c_{i j}$ & $\gamma$ \\
\hline Indoor & $-65 \mathrm{dBm}$ & $-90 \mathrm{dBm}$ & $-40 \mathrm{dBm}$ & $20 \mathrm{~mm}$ \\
\hline Outdoor & $-70 \mathrm{dBm}$ & $-95 \mathrm{dBm}$ & $-50 \mathrm{dBm}$ & $50 \mathrm{~mm}$ \\
\hline
\end{tabular}

\subsection{Indoor and outdoor}

The environmental parameters has been listed in Table 3. Here, $\rho$ means the RSSI threshold of smooth traffic without obvious decaying while $R$ means the link cutoff RSSI of current environment. $c_{i j}$ is the optimal desired RSSI value in our system. For obstacles avoidance, $\gamma$ is designed to denote the safe distance between robots and barriers. When the distance acquired from laser or sonar is larger than $\gamma$, the virtual repulsive force would resist the robot as a result of turning.

\subsection{Link and motion test}

In order to testify the effectiveness of the proposed metric for our control algorithm, a test of robotic cooperation is implemented using real-time sampled link metric. Six robots are deployed in the end of $U$ corridor. First, the team transforms into a chained form network. Then, the team leader is demanded to take exploring along the wall. The speed of leader is set at $110 \mathrm{~mm} / \mathrm{s}$. The desired RSSI value $c_{i j}$ is set as $-40 \mathrm{dBm}$. The parameter $K_{p}$ is set as 25 . The objective is to extend the team of robots so as to maintain the end-end communication link during team leader's 
exploration. The sonar sensors are used to detect the range with wall in order to avoid colliding. Fig. 10 shows the RSSI measurements from every mobile robot. The relationship between RSSI and related distance exhibited the RSSI variety which could act as a feedback for robotic motion. When the RSSI measurement is lager than $-40 \mathrm{dBm}$, which means a repulsive force rather than an abstractive force could be loaded, it could result in speed descending sharply or moving back. For example, five sharp spikes in Fig. 10(a) reflect the robot 2 keep moving forth and back. Note that the speed of each robot varies with respect to the dispersion of desired measurement. This PI control method could guarantee the smooth of robotic motion and cooperative stability.

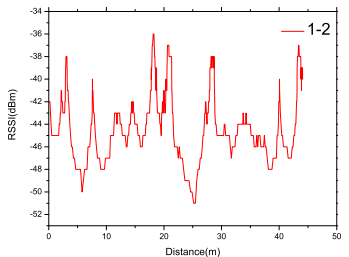

(a) Robot 1 and 2

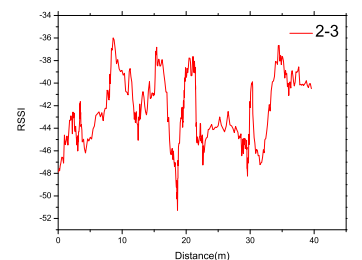

(b) Robot 2 and 3

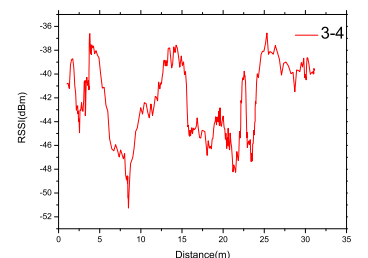

(c) Robot 3 and 4

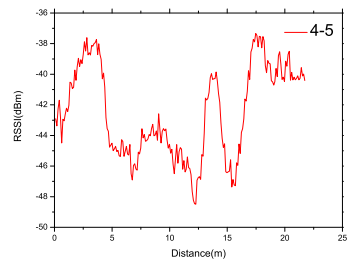

(d) Robot 4 and 5

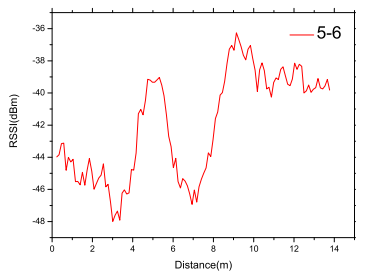

(e) Robot 5 and 6

Figure 10: Experimental results in an $U$ corridor

\section{Conclusions}

A comprehensive metric for evaluating communication link quality and the robotic cooperation algorithms are proposed in this paper. Received signal strength indicator has been testified to be an assessment for link quality by a series of empirical experiments. Throughput and packet loss rate are the complements for the control system by generating critical point and cutoff point respectively. Virtual forces are produced to drive the robot composed of repulsive forces and abstractive forces. Two graphic models including self-healing and tethering are proposed to illustrated the kinematic modes of the team of robots. Experimental results showed the feasibility of our comprehensive metric for link quality and stability of our control schemes.

\section{Bibliography}

[1] E. Stump, A. Jadbabaie, and V. Kumar. Connectivity management in mobile robot teams. Robotics and Automation, 2008. ICRA 2008. IEEE International Conference on, pages 1525-1530, May 2008.

[2] M.R. Souryal, L. Klein-Berndt, L.E. Miller, and N. Moayeri. Link assessment in an indoor 802.11 network. Wireless Communications and Networking Conference, 2006. WCNC 2006. IEEE, 3:1402-1407, 2006. 
[3] Cory Dixon and Eric W. Frew. Maintaining optimal communication chains in robotic sensor networks using mobility control. In Mobile Networks and Applications, volume 14, Issue 3, June 2009 .

[4] K.A. Luthy, E. Grant, and T.C. Henderson. Leveraging rssi for robotic repair of disconnected wireless sensor networks. Robotics and Automation, 2007 IEEE International Conference on, pages 3659-3664, April 2007.

[5] Yongguo Mei, Yung-Hsiang Lu, Y.C. Hu, and C.S.G. Lee. Deployment of mobile robots with energy and timing constraints. Robotics, IEEE Transactions on, 22(3):507-522, June 2006 .

[6] P. Ogren, E. Fiorelli, and N.E. Leonard. Cooperative control of mobile sensor networks:adaptive gradient climbing in a distributed environment. Automatic Control, IEEE Transactions on, 49(8):1292-1302, Aug. 2004.

[7] Jindong Tan. A scalable graph model and coordination algorithms for multi-robot systems. Advanced Intelligent Mechatronics. Proceedings, 2005 IEEE/ASME International Conference on, pages 1529-1534, July 2005.

[8] C. Dixon and E.W. Frew. Controlling the mobility of network nodes using decentralized extremum seeking. Decision and Control, 2006 45th IEEE Conference on, pages 1291-1296, Dec. 2006.

[9] E.W. Frew, D.A. Lawrence, C. Dixon, J. Elston, and W.J. Pisano. Lyapunov guidance vector fields for unmanned aircraft applications. American Control Conference,. ACC '07, pages 371-376, July 2007.

[10] M.R. Souryal and N. Moayeri. Channel-adaptive relaying in mobile ad hoc networks with fading. Sensor and Ad Hoc Communications and Networks, IEEE SECON 2005. Second Annual IEEE Communications Society Conference on, pages 142-152, Sept., 2005.

[11] Michael R. Souryal, Johannes Geissbuehler, Leonard E. Miller, and Nader Moayeri. Realtime deployment of multihop relays for range extension. In MobiSys '0\%: Proceedings of the 5th international conference on Mobile systems, applications and services, pages 85-98, New York, NY, USA, 2007. ACM.

[12] Arzad Kherani Sorav Bansal, Rajeev Shoreyý. Performance of tcp and udp protocols in multi-hop multi-rate wireless networks. In IEEE Wireless Communications and Networking Conference, volume Vol.1, pages 231 - 236, 2004.

[13] Henrik Lundgren, Erik Nordströ, and Christian Tschudin. Coping with communication gray zones in ieee $802.11 \mathrm{~b}$ based ad hoc networks. In WOWMOM '02: Proceedings of the 5th ACM international workshop on Wireless mobile multimedia, pages 49-55, New York, NY, USA, 2002. ACM.

[14] Wei Zhuang, Xi Chen, Jindong Tan, and Aiguo Song. An empirical analysis for evaluating the link quality of robotic sensor networks. In IEEE international conference on wireless communication and signal processing, Nanjing, China, November 13-15, 2009., 2009. 